\title{
Influencia del procesado industrial sobre las características químico-físicas y contenido en lípidos y antioxidantes del salvado de arroz
}

\author{
Por Vanessa R Pestana, ${ }^{a \star}$ Rui C Zambiazi, ${ }^{b}$ Carla R B Mendonça, ${ }^{b}$ Mariângela H Bruscatto, ${ }^{a}$ \\ y Guillermo Ramis-Ramos ${ }^{c}$
}

\author{
a Depto. de La Ciência e Tecnologia Agroindustrial, Universidade Federal de Pelotas, \\ 96010-900 Pelotas, RS, Brasil \\ ${ }^{\text {b }}$ Depto. de Ciência dos Alimentos, Universidade Federal de Pelotas, 96010-900 Pelotas, RS, Brasil \\ c Depto. de Química Analítica, Facultad de Química, Universidad de Valencia, \\ E-46100 Burjassot, Spain. \\ ( ${ }^{\star}$ Autor para correspondencia: vanessapestana@yahoo.com.br)
}

\section{RESUMEN}

Influencia del procesado industrial sobre las características químico-físicas y contenido en lípidos y antioxidantes del salvado de arroz.

Se investigaron las características químico-físicas de los salvados de arroz en distintos momentos de su procesado industrial, incluyendo materia prima (salvados de arroz blanco y vaporizado), salvado peletizado y salvado desgrasado. Se determinó la humedad, la acidez y el índice de peróxidos. Utilizando extractos en éter de petróleo y cromatografía de gases, se estableció el contenido lipídico total y los perfiles de los ácidos grasos. Mediante HPLC se determinaron los tocoferoles y el $\gamma$-orizanol. El salvado de arroz vaporizado mostró la mayor concentración de lípidos y la menor acidez, lo que indica que el vaporizado es el proceso más eficaz para estabilizar el salvado. Los ácidos oleico, linoleico y palmítico fueron los mayoritarios en todas las muestras. El peletizado no produjo pérdidas de lípidos, ni de tocoferoles y $\gamma$-orizanol. Todas las muestras de salvado mostraron contenidos altos de $\alpha$-tocoferol, intermedios de $\gamma$-tocoferol y pequeños de $\delta$-tocoferol. Se detectaron nueve componentes del $\gamma$-orizanol, con una mayor proporción del componente eluido en cuarta posición, probablemente, ferulato de 24metilén cicloartenilo. El salvado peletizado presentó el mayor contenido de tocoferoles, mientras que el de arroz vaporizado mostró el mayor contenido de $\gamma$-orizanol.

PALABRAS-CLAVE: Ácidos grasos - Características químico-físicas - $\gamma$-Orizanol - Procesado del salvado - Salvado de arroz - Tocoferoles.

\section{SUMMARY}

The Influence of Industrial Processing on the Physico-Chemical Characteristics and Lipid and Antioxidant Contents of Rice Bran.

A comparative study of the physico-chemical characteristics of rice bran during the successive steps of its industrial processing was carried out and included white and parboiled rice brans and pelletized and defatted rice brans. Moisture, acidity and peroxide index were determined. Using extracts in petroleum ether and gas chromatography, the total fat contents and the profiles of the fatty acids were established. The tocopherols and $\gamma$-oryzanol were determined using high performance liquid chromatography. The bran of parboiled rice showed the largest fat content and the lowest acidity, indicating that parboiling is the most effective process for bran stabilization. Oleic, linoleic and palmitic acids predominated in all the samples. Pelletization did not produce a loss in lipids, tocopherols or $\gamma$-oryzanol. All the samples showed higher contents of $\alpha$-tocopherol, intermediate contents of $\gamma$-tocopherol and much lower concentrations of $\delta$-tocopherol. Nine components of $\gamma$ oryzanol were detected, with a major proportion of the component that eluted in the fourth position, probably ferulate of 24-methylene cycloartenol. Pelletized rice bran showed the highest tocopherol content, whereas parboiled rice bran yielded the largest $\gamma$-oryzanol content.

KEY-WORDS: Bran processing - Chemical-physical characteristics - Fatty acids - $\gamma$-Oryzanol - Rice bran Tocopherols.

\section{INTRODUCCIÓN}

El salvado de arroz es el subproducto resultante de la etapa de pulido del grano, y representa entre un 8 y un $11 \%$ del peso total del grano entero (PARRADO et al., 2006). El salvado es una excelente fuente de minerales, proteínas y aceite, además de contener un significativo contenido de vitaminas (Carvalho y Vieira, 1999; Parrado et al., 2006). El elevado contenido de lípidos, asociado al bajo valor comercial del salvado, justifican su empleo como materia prima de la industria de extracción y refino de aceites comestibles (Silva, Sanches y Amante, 2001). Sin embargo, la conservación del salvado de arroz es muy problemática, especialmente debido a la acción enzimática de la lipasa. Los ácidos grasos liberados por la hidrólisis de los triglicéridos proporcionan un sabor ácido y desagradable, comprometiendo la utilización del salvado como aditivo alimentario, y como materia prima para la extracción de aceites comestibles. Por esta razón, la temprana inactivación de la lipasa es esencial para estabilizar el salvado, permitiendo su almacenamiento y utilización como mate- 
ria prima alimentaría (Lakkakula, Lima y Walker, 2004). Además, los triglicéridos y otros componentes del aceite se oxidan con facilidad, agregando olores y sabores desagradables, y pérdida de diversos fitoquímicos de interés para la salud humana (Carvalho y Vieira, 1999; Rodrigues, Onoyama y Meirelles, 2006; Rodrigues, Pessoa Filho y Meirelles, 2004; Ausman, Rong y Nicolosi, 2005; Chen y Bergman, 2005; Gavino et al., 2007; Ortoefer, 1996).

En la fracción lipídica del salvado de arroz se encuentran triglicéridos formados mayoritariamente por los ácidos palmítico (12,3-20,5\%), linoleico $(27,0-40,7 \%)$ y oleico $(37,1-52,8 \%)$ (Tortosa y Barber, 1979). Entre los antioxidantes, son de destacar los tocoferoles y tocotrienoles (tocoles), que presentan una importante actividad vitamínica, y los ésteres del ácido trans-ferúlico con esteroles y alcoholes triterpénicos, cuya fracción se conoce como $\gamma$-orizanol (Rodrigues, Onoyama y Meirelles, 2006; Kim et al., 2001; Engel et al, 2003; Fang y Badger, 2003; Juliano et al, 2005).

Entre los tocoles presentes en el salvado de arroz y en el aceite extraído del mismo, predominan $\alpha-, \gamma$ - y $\delta$-tocoferol. Estos compuestos tienen actividad antioxidante y desempeñan un papel protector frente a algunas formas de cáncer (Block y Langseth, 1994; Gliszczynska-Swiglo y Sikorska, 2004; Kamal-Edin y Appelqvist, 1996; Kim et al, 2001). El $\gamma$-orizanol presenta una alta actividad antioxidante, y diversos estudios han demostrado su capacidad para reducir el colesterol plasmático, reducir la absorción del colesterol hepático y prevenir la arteriosclerosis (Wilson et al., 2007; GongYuanssheng y Yao-Huiyuan, 2001; Nyströn et al, 2007; Nyströn et al, 2005).

Para la extracción industrial de aceite se utiliza una mezcla de salvados de arroz blanco (70\%) y vaporizado (30\%). Como se indica en el esquema de la Figura 1, los salvados de arroz blanco y arroz vaporizado proceden del pulido de los arroces integrales no vaporizado y vaporizado, respectivamente. Los arroces integrales se obtienen a partir del arroz "paddy" o bruto, previamente secado y descascarillado. Por su parte, el arroz vaporizado ha sido sometido a un proceso de encharcamiento y tratamiento en autoclave antes del secado y descascarillado. En ambos casos, el pulido final del grano origina el salvado como subproducto.

Antes de proceder a extraer el aceite, la mezcla de salvados se somete a un proceso de acondicionado, que consiste en una etapa de cocido para inactivar las enzimas, seguida de otra de aglomerado o peletizado, que facilita la extracción. Después de extraer el aceite mediante un disolvente, se obtiene el salvado de arroz desgrasado, que normalmente es aprovechado para la elaboración de piensos.

Las etapas de acondicionado pueden provocar alteraciones en los ácidos grasos del salvado de arroz, y pérdidas de importantes antioxidantes, como tocoferoles y $\gamma$-orizanol. El estudio de la posible alteración de estos compuestos durante el acondicionado tiene interés desde los puntos de vista

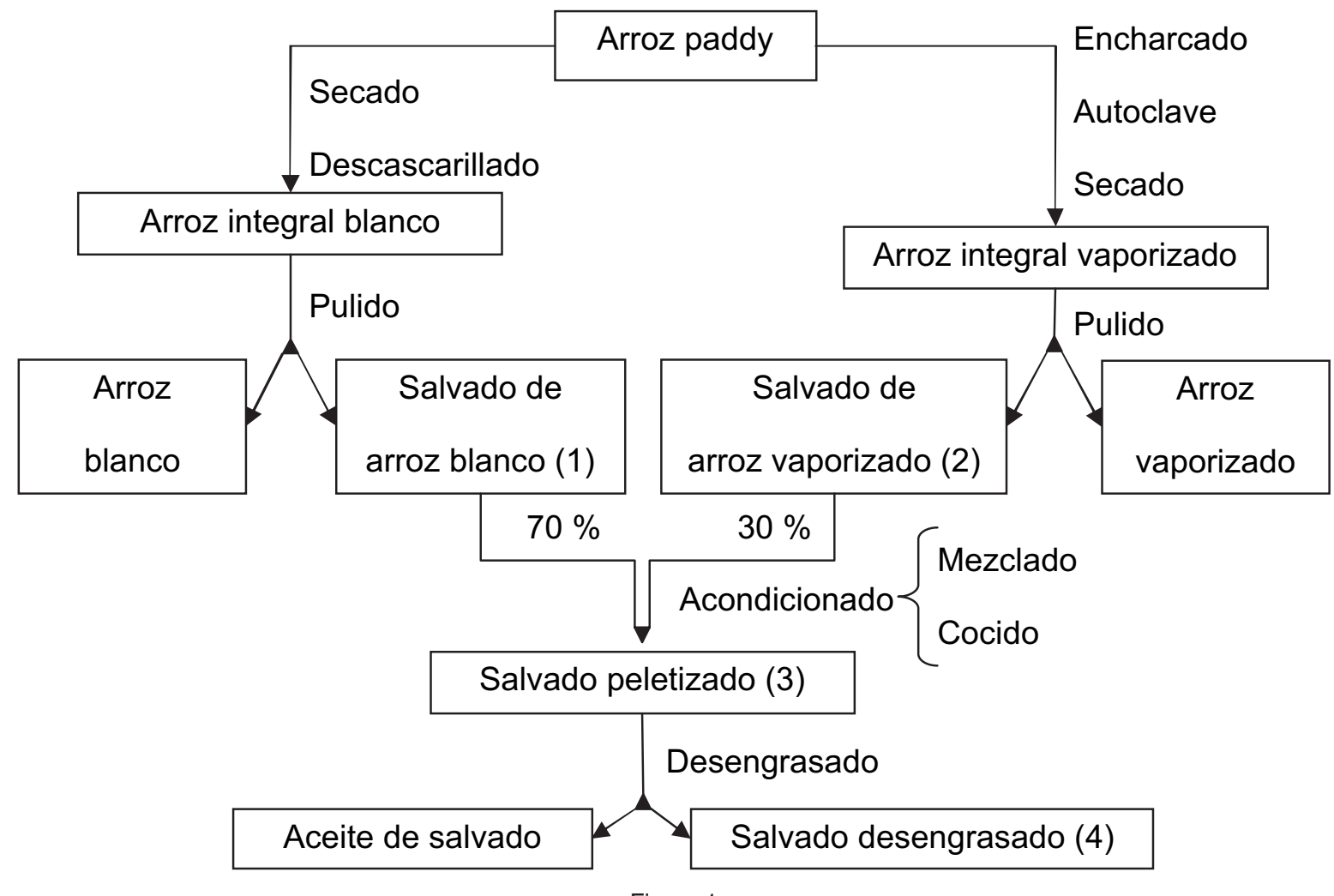

Figura 1

Esquema del procesado industrial del salvado de arroz.

Los números entre paréntesis indican los tipos de salvado estudiados en este trabajo. 
industrial y nutricional. El objetivo de este trabajo fue evaluar los efectos del acondicionado y desgrasado de los salvados de arroz blanco y vaporizado a lo largo de todas las etapas de su procesado industrial. Mediante el estudio pormenorizado de la alteración de las características químico-físicas, composición en ácidos grasos, y contenidos de tocoferoles y de $\gamma$ orizanol, en cada etapa, se pretende conseguir una visión a la vez detallada y global de sus efectos.

\section{MATERIALES Y MÉTODOS}

\subsection{Materias primas}

Las muestras de salvado de arroz fueron donadas por una industria productora de aceite de arroz (Industria Rio-Grandense de Óleos Vegetais LTDAIrgovel, Pelotas, RS, Brasil). Las muestras de los distintos tipos de salvado se recogieron directamente de la línea de procesado de la referida industria. Dentro del esquema de procesado del salvado de la Figura 1, las distintas muestras recogidas se indican con los números 1 a 4 . Se recogieron muestras de los salvados de arroz blanco (1) y de arroz vaporizado (2), y de los salvados peletizado (3) y desgrasado (4). Las muestras se recogieron a partir de tres lotes distintos de materia prima. Las muestras se almacenaron $\mathrm{a}-18^{\circ} \mathrm{C}$ hasta el momento de los análisis. Para cada lote se realizaron análisis duplicados. Por ello, cada dato presentado en este trabajo se obtuvo como promedio de seis medidas $(n=6)$.

\subsection{Procedimientos}

\section{Caracterización químico-física}

Las muestras de salvado se caracterizaron mediante la determinación del extracto en éter, contenido en humedad, e índices de acidez y de peróxidos, siguiendo la metodología descrita por la AOCS (1992). Para determinar los índices de acidez y peróxidos, se tomaron muestras de 2 y $5 \mathrm{~g}$ respectivamente, y se extrajeron durante unas $15 \mathrm{~h}$ con mezclas de disolventes. Para medir el índice de acidez se utilizó una proporción 2:10 de salvado/ disolvente $(p / v)$, estando este último constituido por éter dietílico/ etanol en proporción 2:1 (v/v). Después de la extracción, se filtró a través de papel filtro, y el filtrado se aforó a $25 \mathrm{~mL}$ con la misma mezcla de éter dietílico y etanol. Para medir el índice de peróxidos se utilizó una proporción 1:6 de salvado/ disolvente $(\mathrm{p} / \mathrm{v})$, estando este último constituido por una mezcla de ácido acético/ cloroformo en proporción 3:2 (v/v). El filtrado se aforó a $30 \mathrm{~mL}$ con la misma mezcla de disolventes. Los extractos se utilizaron para determinar los índices de acidez y peróxidos.

\section{Determinación de ácidos grasos mediante cromatografía de gases}

Para determinar la composición en ácidos grasos se utilizó un cromatógrafo de gases Shimadzu
GC-14B (Kyoto, Japón), provisto de un detector de ionización de llama (FID), y de una columna capilar ( $\mathrm{J} \&$ W Scientific, Folsom, CA, USA) de $30 \mathrm{~m} \times 0,25$ $\mathrm{mm}$, cubierta por una fase líquida DB-2250 de 25 $\mu \mathrm{m}$. Los datos se guardaron y procesaron con auxilio del software Class GC10 (Shimadzu).

Se pesaron unos $10 \mathrm{~g}$ de salvado, y se sometieron a extracción Soxhlet con éter de petróleo durante $4 \mathrm{~h}$. Los extractos se centrifugaron a $3000 \mathrm{rpm}$ durante $10 \mathrm{~min}$, y se evaporó el disolvente a $50^{\circ} \mathrm{C}$ utilizando un rotavapor. Se tomaron porciones de unos $45 \mathrm{mg}$ de los distintos aceites extraídos, se introdujeron en tubos de ensayo y se adicionó $1 \mathrm{~mL}$ de éter de petróleo y $12 \mathrm{~mL}$ de $\mathrm{HCl} 0,5 \mathrm{~N}$ en metanol. Se taparon los tubos, se agitaron con un vortex y se calentaron en estufa a $65^{\circ} \mathrm{C}$ durante $1 \mathrm{~h}$. Después de enfriar se adicionaron $5 \mathrm{~mL}$ de isooctano y $6 \mathrm{~mL}$ de agua destilada, se agitó vigorosamente y se dejó decantar. Se inyectaron $1,5 \mu \mathrm{L}$ de la capa superior en el cromatógrafo de gases.

Para la separación de los ácidos grasos se programó una etapa inicial a $100^{\circ} \mathrm{C}$ durante $5 \mathrm{~min}$, seguida de una rampa lineal de $8^{\circ} \mathrm{C} \mathrm{min}^{-1}$ hasta alcanzar $150^{\circ} \mathrm{C}$. Esta temperatura se mantuvo durante $5 \mathrm{~min}$, y luego se aumentó hasta $180^{\circ} \mathrm{C}$ mediante una rampa lineal de $1,5^{\circ} \mathrm{C} \mathrm{min}^{-1}$. Después de 5 min se volvió a aumentar hasta $220^{\circ} \mathrm{C}$ mediante otra rampa lineal de $2^{\circ} \mathrm{C} \mathrm{min}^{-1}$. Esta última temperatura se mantuvo durante $6 \mathrm{~min}$, siendo el tiempo total de separación de 58,25 min. $\mathrm{El}$ inyector y el detector se mantuvieron a $250^{\circ} \mathrm{C}$. Se utilizó nitrógeno como gas portador a un caudal de $1,0 \mathrm{~mL} \mathrm{~min}^{-1}$ (Zambiazi et al., 2007).

Para la identificación de los ácidos grasos se utilizó una mezcla de estándares conteniendo los ácidos caproico (C6:0), caprílico (C8:0), cáprico (C10:0), caproleico (C10:1), láurico $(C 12: 0)$, dodecenoico (C12:1), mirístico (C14:0), miristoleico (C14:1), palmítico (C16:0), palmitoleico (C16:1), margárico (C17:0), heptadecenoico (C17:1), esteárico (C18:0), oleico (C18:1), linoleico (C18:2), linolénico (C18:3), araquídico (C20:0), gadoleico (C20:1), eicosadienoico (C20:2), eicosatrienoico (C22:3), eicosatetraenoico (C22:4), behénico (C22:0), erúcico (C22:1), docosadienoico (C22:2), docosatrienoico (C22:3), docosatetraenoico (C22:4), lignocérico (C24:0) y nervónico (C24:1) (Sigma-Aldrich, Steinheim, Alemania).

\section{Determinación de tocoferoles y $\gamma$-orizanol mediante cromatografía líquida}

La determinación de tocoferoles y $\gamma$-orizanol se llevó a cabo sobre los mismos extractos obtenidos para determinar ácidos grasos. Alícuotas de unos $500 \mathrm{mg}$ se diluyeron hasta $5 \mathrm{~mL}$ con una mezcla isopropanol/ acetonitrilo (7:3, v/v). Se centrifugó durante 6 min a 9000 rpm (microcentrífuga NT800, Nova Técnica, Piracicaba, SP, Brasil), y se inyectaron alícuotas del sobrenadante en el cromatógrafo líquido. Se utilizó un cromatógrafo de líquidos de alta eficacia (Shimadzu), constituido por un módulo 
de mezcla de disolventes (LC-10ATVP), desgasificador (FCV-10ALVP), bomba cuaternaria (Reodyne DGU-14A), sistema de control (SCL-10AVP), horno de columnas (CTO-10ASVP), e inyector automático (SIL-10AF). Se utilizó una columna analítica de fase inversa Shim-Pak CLC-ODS (octadecilsílica, 4 $\mu \mathrm{m}, 39 \mathrm{~mm} \times 150 \mathrm{~mm}$ ). Se inyectaron alícuotas de 20 y $40 \mu \mathrm{L}$, y las separaciones se llevaron a cabo a $25^{\circ} \mathrm{C}$ y con un caudal de $1 \mathrm{~mL} \min -1$. El $\gamma$-orizanol se monitorizó a $325 \mathrm{~nm}$ mediante un detector espectrofotométrico UV-vis (SPD-10AVVP). Los tocoferoles se monitorizaron mediante un detector fluorimétrico (RF-10AXL), utilizando 290 y $330 \mathrm{~nm}$ como longitudes de onda de excitación y emisión, respectivamente. Los datos se guardaron y procesaron con el software Class-VP (Shimadzu).

Para el análisis de tocoferoles, la fase móvil inicial fue acetonitrilo/ metanol/ isopropanol en proporción 50:40:10 (v/v/v) durante $10 \mathrm{~min}$. A continuación, mediante una rampa lineal de 2 min se pasó a una fase de acetonitrilo/ metanol/ isopropanol en proporción 30:65:5 (v/v/v). Esta fase se mantuvo durante $12 \mathrm{~min}$, y se regresó a la fase móvil inicial, siendo el tiempo total de separación de $15 \mathrm{~min}$.

Para el análisis de $\gamma$-orizanol, se utilizó una fase móvil inicial de acetonitrilo/ metanol/ isopropanol en proporción 50:40:10 (v/v/v) durante $10 \mathrm{~min}$. A continuación, mediante una rampa lineal de $15 \mathrm{~min}$, se pasó a una fase conteniendo acetonitrilo/ metanol/ isopropanol en proporción 30:65:5 (v/v/v). Después de 5 min se regresó a la fase móvil inicial, de modo que el tiempo total del desarrollo cromatográfico fue de $30 \mathrm{~min}$. Se construyeron curvas de calibración externa para $\delta$-tocoferol (Sigma, $90 \%$ ), $\gamma$-tocoferol (Sigma, $\geq 96 \%$ ), $\alpha$-tocoferol (Merck, 99\%) y $\gamma$ orizanol (pureza analítica, TCI, Tokyo, Japón).

Con vistas a identificar los componentes del $\gamma$ orizanol, las fracciones separadas por HPLC se recogieron manualmente en viales. Alícuotas de cada fracción se inyectaron en un espectrómetro de masas con trampa de iones (ITMS), provisto de una fuente de ionización electrospray (ESI) (1100 Series, Agilent, Waldbronn, Alemania), através de un capilar de sílice fundida con $50 \mu \mathrm{m}$ d.i. (Supelco, Bellefonte, PA, USA). Para ese propósito se utilizó una bomba de jeringa (kd Scientific, Holliston, MA, USA) que suministró un flujo constante de $0.3 \mathrm{ml}$ $\mathrm{h}^{-1}\left(5 \mu \mathrm{l} \mathrm{min}^{-1}\right)$. Las condicciones de trabajo en ITMS fueron: presión de gas en el nebulizador, 25

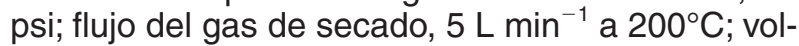

taje del capilar, $3.5 \mathrm{kV}$; voltajes de las máscaras 1 y $2,-26.8 \mathrm{~V}$ y $-6.0 \mathrm{~V}$, respectivamente. Se usó nitrógeno como gas de secado y nebulización (generador Gaslab NG LCMS 20, Equcien, Madrid, España). El espectro de masas se barrió en el rango $\mathrm{m} / \mathrm{z}$ 100-800 en modo ion negativo. Como masa objetivo se utilizó $\mathrm{m} / \mathrm{z} 601$ (pico $[\mathrm{M}-\mathrm{H}]^{-}$del cicloartenilferulato). La máxima carga de la trampa de iones se estableció en $3 \times 10^{4}$ cuentas, y el tiempo máximo de registro fue $300 \mathrm{~ms}$.

\subsection{Análisis estadístico}

Se realizó un análisis de la varianza (ANOVA) de los resultados, y se compararon las medias mediante el test de Tukey, aplicando en todos los casos un nivel de significación del $5 \%$. Para ello, se utilizó el paquete estadístico Statistica vs. 6.0 (StatSoft, 2004).

\section{RESULTADOS Y DISCUSIÓN}

\subsection{Caracterización químico-física de los salvados de arroz}

\section{Contenido en humedad}

En la Tabla 1 se muestran los valores del contenido en humedad y demás parámetros físico-químicos obtenidos con los salvados de arroz blanco, vaporizado, peletizado y desgrasado. El contenido en humedad del salvado varió significativamente a lo largo de las etapas de procesado estudiadas. El mayor contenido en humedad se encontró en el salvado de arroz desgrasado. Hwang et al. (2005) encontraron un contenido de humedad para salvado de arroz entre el 9,6 y el $14,7 \%$. Otros estudios indican valores de humedad del 8,0 y del $10,0 \%$ para salvado de arroz blanco y de arroz vaporizado, respectivamente (Silva, Sanches y Amante, 2006). Los valores de la Tabla 1 son similares a los indicados en la bibliografía, si bien, se encontró una mayor humedad en el arroz blanco que en el vaporizado. Este resultado puede explicarse por la distinta intensidad de los procesos previos de secado en cada industria en particular. Igualmente, la humedad intermedia del salvado peletizado se explica por proceder éste de una mezcla de los salvados de arroz blanco y vaporizado. Finalmente, la

Tabla 1

Características químico-físicas de los salvados de arroz ${ }^{a}$

\begin{tabular}{lcccc}
\hline $\begin{array}{c}\text { Tipo de } \\
\text { salvado }\end{array}$ & $\begin{array}{c}\text { Humedad } \\
\mathbf{( \% )}\end{array}$ & $\begin{array}{c}\text { Extracto } \\
\text { en éter }(\%)\end{array}$ & $\begin{array}{c}\text { Acidez } \\
\mathbf{( \%} \text { ác. oleico) }\end{array}$ & $\begin{array}{c}\text { Indice de peróxido } \\
\text { (meq-g kg-1) }\end{array}$ \\
\hline Arroz blanco & $10,6 \mathrm{a}$ & $19,5 \mathrm{a}$ & $1,40 \mathrm{a}$ & No detectado \\
Arroz vaporizado & $8,9 \mathrm{~b}$ & $23,4 \mathrm{~b}$ & $0,64 \mathrm{~b}$ & $1,22 \mathrm{a}$ \\
Peletizado & $9,6 \mathrm{c}$ & $19,2 \mathrm{a}$ & $1,52 \mathrm{a}$ & $1,11 \mathrm{a}$ \\
Desgrasado & $12,8 \mathrm{~d}$ & $0,13 \mathrm{c}$ & $0,40 \mathrm{~b}$ & No detectado \\
\hline
\end{tabular}

a Para cada dato de la tabla, $n=6$. En una misma columna, letras distintas indican diferencia significativa al nivel del $5 \%$, según el test de Tukey $(p<0,05$, . 
mayor humedad del salvado desgrasado puede explicarse por la pérdida de peso del mismo durante la extracción del aceite.

\section{Extracto en éter}

El salvado de arroz vaporizado presentó el mayor valor del extracto en éter. Teniendo en cuenta las diferencias de humedad de las muestras, se calculó el valor del extracto en éter respecto a muestra seca. Se verificó así que el salvado de arroz vaporizado presentaba una concentración de lípidos cerca de un 15\% superior a la de los salvados de arroz blanco y peletizado.

En concordancia con estos resultados, se han informado valores del extracto en éter del $27,8 \%$ para salvado de arroz vaporizado, y del $18,5 \%$ para salvado de arroz blanco (Denardin et al., 2003). El arroz vaporizado presenta una mayor consistencia física que el arroz no vaporizado. Como es fácil de comprobar, el arroz vaporizado es más resistente a la abrasión. Es por ello que, a pesar de la acción más enérgica de los bruñidores durante el pulido, resulta un salvado con una mayor proporción de capas externas y, por ello, con una menor proporción de endospermo. La presencia de mayor proporción de endospermo en el salvado de arroz blanco produce un efecto de "dilución" del extracto en éter en el mismo.

Igualmente en relación con el extracto en éter, no se observaron diferencias significativas entre los salvados de arroz blanco y peletizado (calculados respecto a muestra seca). Este resultado se explica porque en el salvado peletizado predomina el salvado de arroz blanco (70\%). Por su parte, el menor contenido de extracto en éter en el salvado desgrasado está obviamente relacionado con la extracción previa del aceite por medio de disolventes. Se ha indicado que el salvado resultante de un proceso eficiente de extracción del aceite debe contener menos del $1 \%$ de extracto en éter (Rittner, 1999).

\section{Índice de acidez}

El salvado de arroz blanco presentó un índice de acidez mayor que el salvado de arroz vaporizado. Se sabe que la actividad de la lipasa presente en el salvado de arroz blanco es responsable de la hidrólisis de los triglicéridos, con la consiguiente liberación de ácidos grasos, lo que origina un aumento de la acidez (Denardin et al., 2003). El vaporizado desactiva las enzimas, por lo que estabiliza el salvado de arroz. En la bibliografía se indican valores de acidez soluble en alcohol del 1,14\% para el salvado de arroz vaporizado, y del $15,4 \%$ para el salvado de arroz blanco (Silva, Sanches y Amante, 2006).

Los valores de acidez obtenidos para los salvados de arroz blanco y peletizado no fueron significativamente diferentes entre ellos. Sin embargo, el salvado de arroz peletizado mostró una tendencia a tener un mayor índice de acidez que el salvado de arroz blanco. Probablemente, esta diferencia es debida al tiempo transcurrido durante el mezclado de los salvados de arroz blanco y vaporizado, y las etapas de acondicionado (cocido y peletizado). El proceso de acondicionado puede contribuir al aumento de la acidez del salvado por permitir la acción de la lipasa.

Por otra parte, los salvados de arroz vaporizado y desgrasado mostraron distintos valores de acidez, si bien la diferencia no alcanzó el nivel de significación del 5\% (test de Tukey). El bajo contenido en acidez del salvado desgrasado puede ser debido a la eliminación de ácidos grasos libres durante el proceso de extracción con disolventes.

\section{Índice de peróxidos}

Todos los salvados presentaron niveles muy bajos de peróxidos. Tanto en el salvado de arroz vaporizado como en el resultante del proceso de acondicionado (salvado peletizado), la utilización de altas temperaturas podría justificar las pequeñas cantidades de peróxidos observadas. Para el salvado desgrasado, el resultado, por debajo del límite de detección, puede ser atribuido a la eliminación de los peróxidos durante la extracción con disolventes.

\subsection{Composición en ácidos grasos}

La composición en ácidos grasos de los salvados de arroz blanco y vaporizado, y de los salvados peletizado y desgrasado, se presenta en la Tabla 2. Los ácidos grasos encontrados en mayor proporción fueron el oleico $(32,8-35,6 \%)$, linoleico $(30,8-$ $33,5 \%)$ y palmítico $(19,5-21,1 \%)$. Los ácidos grasos saturados e insaturados totales se encontraron en los intervalos $26,6-28,1 \%$ y $71,9-73,4 \%$, respectivamente. El mayor porcentaje de ácidos grasos insaturados es el característico para aceites de gramíneas, y para una gran mayoría de otros aceites vegetales comestibles.

Los datos de la Tabla 2 indican una composición muy similar de los triglicéridos presentes en los salvados de arroz blanco y vaporizado. Dicha composición se mantuvo esencialmente constante a lo largo del procesado del salvado. En presencia de procesos de oxidación se hubiera reducido el porcentaje de ácidos grasos insaturados, resultando aumentado el de los ácidos grasos saturados; sin embargo, este proceso no se observó en este estudio. Entre los ácidos grasos presentes en el salvado de arroz, es interesante observar la presencia de ácido margárico, con una concentración que varió en el rango 0,55-1,35\% del contenido de lípidos. Kitta et al (2005) reportaron la presencia de ese ácido en arroz integral.

\subsection{Identificación y cuantificación de los tocoferoles}

En la Figura $2 \mathrm{~A}$ se muestra un cromatograma típico de una muestra de salvado en el que se observan los picos de $\alpha$-, $\gamma$ - y $\delta$-tocoferol. Los picos de los grupos 1 y 2 de la Figura $2 \mathrm{~A}$ no fueron iden- 
Tabla 2

Perfiles de ácidos grasos en salvados de arroz (\%, como ésteres metílicos)

\begin{tabular}{ccccc}
\hline Ácido graso & Arroz blanco & Arroz vaporizado & Peletizado & Desgrasado \\
\hline C10:0 & Trazas & Trazas & 0,04 & Trazas \\
C12:0 & Trazas & Trazas & 0,07 & 0,09 \\
C14:0 & Trazas & 0,29 & 0,29 & 0,41 \\
C14:1 & 0,24 & Trazas & 0,05 & 2,59 \\
C16:0 & 19,5 & 20,4 & 0,4 & 0,15 \\
C16:1 & 0,15 & 0,15 & 0,55 & 1,19 \\
C17:0 & 1,35 & 0,92 & Trazas & 1,61 \\
C17:1 & Trazas & Trazas & 37,7 & 32,8 \\
C18:0 & 1,53 & 1,74 & 33,3 & 33,5 \\
C18:1 & 36,3 & 36,3 & 2,49 & 4,05 \\
C18:2 & 30,8 & 32,9 & 0,54 & 0,42 \\
C18:3 & 3,72 & 3,01 & 0,50 & 0,66 \\
C20:1 & 0,46 & 0,47 & 0,81 & 1,09 \\
C20:2 & 0,72 & 1,58 & 0,83 & 0,81 \\
C22:0 & 2,08 & 0,99 & Trazas & Trazas \\
C22:2 & 1,59 & Trazas & Trazas & Trazas \\
C22:3 & Trazas & Trazas & 0,50 \\
C22:4 & Trazas & Trazas & 0,44 & 0,66 \\
C24:0 & Trazas & 0,74 & 0,35 & 0,37 \\
C24:1 & 1,19 & 0,30 & 26,7 & 26,6 \\
\hline Saturados & 0,35 & 27,7 & 73,3 & 73,4 \\
\hline Insaturados & 28,1 & 72,3 & & \\
\hline
\end{tabular}

tificados en este estudio, pudiendo tratarse, al menos en parte, de proteínas. En condiciones cromatográficas semejantes a las aquí utilizadas, el orden de elución esperado para tocoferoles (tiempos de retención crecientes) es: $\delta$-tocoferol $<$ $\beta$-tocoferol $\approx \gamma$-tocoferol $<\alpha$-tocoferol (Abidi, 2000). Los picos de $\delta$-tocoferol (pico 3), $\gamma$-tocoferol (pico 4) y $\alpha$-tocoferol (pico 5) aparecieron bien resueltos entre sí y respecto a los picos adyacentes. Las colas de los picos del $\gamma$ - y $\alpha$-tocoferol podrían deberse a los correspondientes tocotrienoles.

La polaridad de los tocoferoles aumenta en el orden: $\alpha<\beta=\gamma<\delta$, esto es, a medida que disminuye de tres a uno el número de grupos metilo unidos al grupo cromanol. La polaridad de $\beta$ - y $\gamma$-tocoferol es muy similar, lo que es debido a que la única diferencia estructural entre ellos es la posición de los dos grupos metilo. Por esta razón, la resolución de este par de compuestos es difícil (Zambiazi, 1997). Además, el $\beta$-tocoferol está presente en cantidades muy pequeñas en salvado de arroz, lo que dificulta aún más su detección frente a concentraciones considerablemente mayores de $\gamma$-tocoferol. Los contenidos de tocoferoles encontrados en los distintos salvados de arroz se muestran en la Tabla 3.

Los contenidos de $\alpha$-tocoferol y de $\gamma$-tocoferol resultaron ser significativamente mayores en los salvados de arroz blanco y peletizado. Por su parte, el contenido de $\delta$-tocoferol fue significativamente mayor en los salvados de arroz vaporizado y peletizado, si bien, en todos los casos las concentraciones de este compuesto fueron muy pequeñas.

Comparando los contenidos totales de tocoferoles en los salvados peletizado y desgrasado, se deduce que la etapa de extracción del aceite reduce el contenido total de tocoferoles en un $91,1 \%$ (de 7,35 a 0,66 mg/ $100 \mathrm{~g}$ ), esto es, la extracción del aceite con disolventes elimina casi la totalidad de los tocoferoles.

También se observó que el contenido total de tocoferoles es un $68,2 \%$ menor en el salvado de arroz vaporizado que en el salvado de arroz blanco.

Tabla 3

Concentraciones de tocoferoles en salvados de arroz, ${ }^{a} \mathrm{mg} / 100 \mathrm{~g}$

\begin{tabular}{|c|c|c|c|c|c|c|c|}
\hline \multirow[b]{2}{*}{ Tipo de salvado } & \multicolumn{2}{|c|}{$\alpha$-Tocoferol } & \multicolumn{2}{|c|}{$\gamma$-Tocoferol } & \multicolumn{2}{|c|}{$\delta$-Tocoferol } & \multirow{2}{*}{$\begin{array}{c}\text { Totales } \\
\mathrm{mg} \cdot 100 \mathrm{~g}^{-1}\end{array}$} \\
\hline & $\mathrm{mg} \cdot 100 \mathrm{~g}^{-1}$ & $\% *$ & $\mathrm{mg} \cdot 100 \mathrm{~g}^{-1}$ & $\%^{*}$ & $\mathrm{mg} \cdot 100 \mathrm{~g}^{-1}$ & $\%^{*}$ & \\
\hline Arroz blanco & $6,21 \mathrm{a}$ & 79,5 & $1,50 \mathrm{a}$ & 19,5 & $0,08 \mathrm{a}$ & 0,98 & 7,82 \\
\hline Arroz vaporizado & $1,84 \mathrm{~b}$ & 74,2 & $0,53 b$ & 21,5 & $0,11 b$ & 4,31 & 2,48 \\
\hline Peletizado & $5,79 a$ & 78,7 & $1,45 \mathrm{a}$ & 19,8 & $0,11 \mathrm{~b}$ & 1,46 & 7,35 \\
\hline Desgrasado & $0,52 \mathrm{~b}$ & 79,3 & $0,12 \mathrm{~b}$ & 18,6 & $0,01 \mathrm{c}$ & 2,13 & 0,66 \\
\hline
\end{tabular}

\footnotetext{
${ }^{a}$ Para cada dato de la tabla, $n=6$. En una misma columna (mg. $\left.100 \mathrm{~g}^{-1}\right)$, letras distintas indican diferencia significativa al nivel del $5 \%$,
} según el test de Tukey $(p<0,05)$.

*Distribución relativa de los tocoferoles en salvados de arroz. 

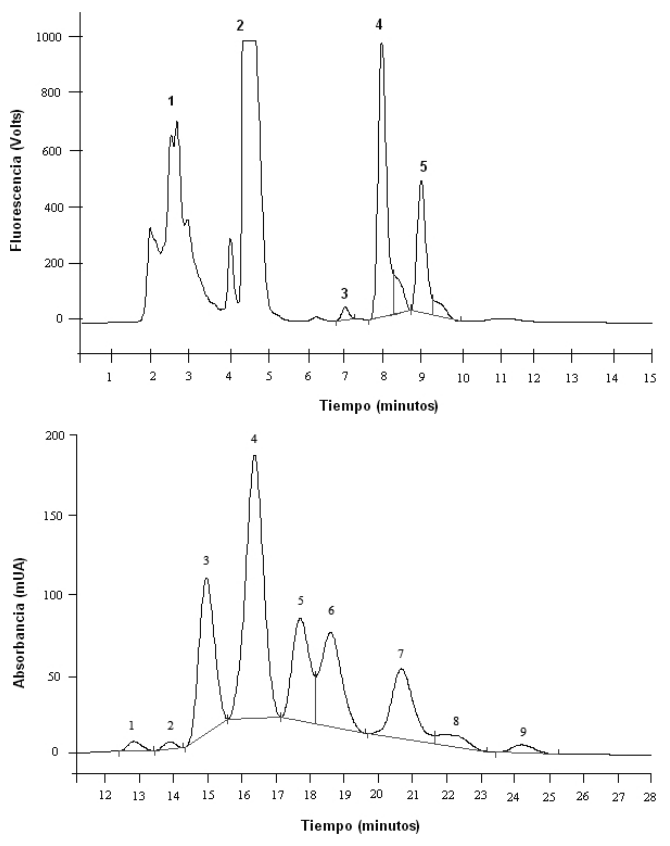

Figura 2

Cromatogramas característicos mostrando los picos de los tocoferoles (A) y del $\gamma$-orizanol (B) en una muestra de salvado de arroz. En A, los picos de los grupos 1 y 2 no fueron identificados; otros picos son: $\delta$-tocoferol (3), $\gamma$-tocoferol (4) y $\alpha$-tocoferol (5). En B, los nueve picos observados no se identificaron en este estudio.

Esta pérdida puede producirse por lixiviación durante el encharcamiento, o por oxidación a causa de las altas temperaturas utilizadas durante el encharcamiento y el posterior tratamiento en autoclave.

Entre los tocoferoles individuales, el $\alpha$-tocoferol se encontró en mayor concentración en todas las muestras, contribuyendo un $74-79 \%$, seguido por $\gamma$ tocoferol $(19-21 \%)$ y por $\delta$-tocoferol $(1-4 \%)$. La menor concentración de $\alpha$-tocoferol se encontró en el salvado de arroz vaporizado (Tabla 3).

Según la bibliografía, la concentración de $\alpha$ tocoferol en el salvado de arroz blanco suele variar entre 2,94 y $8,09 \mathrm{mg} / 100 \mathrm{~g}$, dependiendo de las diferencias entre variedades de arroz (Chen y Bergman, 2005). Gavino et al. (2007), investigando salvado de arroz blanco procedente de diferentes variedades de arroz, encontraron tocoferoles totales en el rango 4,1-6,1 mg/ $100 \mathrm{~g}$. Estas concentraciones son similares a las encontradas en este estudio (7,82 mg/ $100 \mathrm{~g})$.

\subsection{Identificación y cuantificación de $\gamma$-orizanol}

En la Figura 3 se muestra un cromatograma típico de la fracción de $\gamma$-orizanol en las muestras de salvado de arroz. Se observan hasta nueve picos, cinco de ellos perfectamente resueltos. No se identificaron componentes individuales del $\gamma$-orizanol por no disponer de estándares individuales de los mismos. Xu y Godber (1999) llevaron a cabo una separación cromatográfica en condiciones muy similares a las empleadas en este estudio, identificando los picos mediante espectrometría de masas. Comparando los resultados obtenidos con los descritos por Xu y Godber, se deduce que los picos 1-9 de la Figura $2 \mathrm{~B}$ corresponden a ferulato de $\Delta^{7}$-estigmastenilo $\left(1, t_{R}=12,9 \mathrm{~min}\right)$, ferulato de estigmasterilo $\left(2, t_{R}=13,9 \mathrm{~min}\right)$, ferulato de cicloartenilo $\left(3, t_{R}=15,0 \mathrm{~min}\right)$, ferulato de 24-metiléncicloartenilo $\left(4, t_{R}=16,5 \mathrm{~min}\right)$, mezcla de ferulato de $\Delta^{7}$-campestenilo, ferulato de campesterilo y ferulato de $\Delta^{7}$-sitostenilo (5 y $6, t_{R}$ de 17,9 a 18,0 min), ferulato de sitosterilo $\left(7, t_{R}=20,7 \mathrm{~min}\right)$, ferulato de campestanilo $\left(8, t_{R}=22,0 \mathrm{~min}\right)$ y ferulato de sitostanilo $\left(9, t_{R}=24,3 \mathrm{~min}\right)$. Los datos de la identificación del $\gamma$-orizanol se muestran en la Tabla 4.

Los contenidos de $\gamma$-orizanol, tanto los globales como los de sus componentes, se estimaron de acuerdo con la recta de calibrado construida con $\gamma$ orizanol comercial (suma de las áreas de todos los picos de los cromatogramas). El espectro de masas del $\gamma$-orizanol comercial mostró los picos predominantes de los iones $[\mathrm{M}-\mathrm{H}]^{-}$de los siguientes ferulatos (en orden decreciente de abundancias): 24metilén-cicloartanilferulato $(\mathrm{m} / \mathrm{z} 615.4)>$ cicloartenilferulato $(\mathrm{m} / \mathrm{z} 601.4)>$ [campesterilferulato $+\Delta^{7}$ campestenil ferulato] $(\mathrm{m} / \mathrm{z} 575.4)>$ [ $\beta$-sitosterilferulato $+\Delta^{7}$-Sitostenil ferulato] $(m / z 589.4)$. Debido a que se utilizó esta mezcla de ferulatos de fitoesteroles como único estándar de calibración, las concentraciones obtenidas deben considerarse como aproximadas. Sin embargo, los errores sistemáticos asociados a los contenidos globales de $\gamma$-orizanol son probablemente muy pequeños, ya que la composición del $\gamma$-orizanol comercial utilizado como estándar resultó ser muy similar a la composición del $\gamma$-orizanol en las muestras. Por otra parte, los errores sistemáticos asociados a la estimación de los componentes individuales de $\gamma$-orizanol no son probablemente mucho mayores que los cometidos

Tabla 4

Identificación de los componentes del $\gamma$-orizanol

\begin{tabular}{|c|c|c|c|}
\hline Pico n. $^{\text {oa }}$ & Tiempo de retención, min & Pico $m / z$ de $[M-H]^{-}$ & Compuesto \\
\hline 3 & 15,1 & 601 & Cicloartenil ferulato \\
\hline 4 & 16,5 & 615 & 24-Metileno cicloartenil ferulato \\
\hline 5 & 17,8 & 575 & $\Delta^{7}$-Campestenil ferulato \\
\hline 6 & 18,7 & 575 & Campesteril ferulato \\
\hline 7 & 20,8 & 589 & $\Delta^{7}$-Sitostenil ferulato \\
\hline 8 & 22,3 & 589 & $\beta$-Sitosteril ferulato \\
\hline 9 & 24,3 & 591 & Sitostenil ferulato \\
\hline
\end{tabular}

\footnotetext{
${ }^{a}$ Según la numeración de los picos en la Fig. 2B.
} 
en la estimación del $\gamma$-orizanol total, ya que las absortividades molares de los ferulatos de estos fitoesteroles a $334 \mathrm{~nm}$ dependen fundamentalmente del grupo ferulato, mostrando además las masas moleculares de estos compuestos diferencias menores que un $7 \%$ en todos los casos. Finalmente, la posible presencia de errores sistemáticos no invalida las conclusiones relacionadas con las variaciones relativas de concentración a lo largo de las etapas del procesado del salvado.

Los contenidos totales de $\gamma$-orizanol en los distintos tipos de salvado estudiados se muestran en la Tabla 5. El mayor contenido de $\gamma$-orizanol se encontró en el salvado de arroz vaporizado, mientras que el salvado de arroz blanco presentó una concentración mucho menor. Esta situación es opuesta a la observada con los tocoferoles (Tabla 3), lo que puede ser debido a una mayor estabilidad del $\gamma$-orizanol a temperaturas altas, o también a su menor solubilidad en agua, por lo que no se perdería durante las etapas de encharcamiento y tratamiento en autoclave a que es sometido el arroz vaporizado.

Aunque la diferencia no es estadísticamente significativa, el contenido de $\gamma$-orizanol en el salvado peletizado fue mayor que en el salvado de arroz blanco. Esta tendencia puede ser debida a la presencia de un $30 \%$ de salvado de arroz vaporizado en la composición del salvado peletizado.

En comparación con los contenidos de tocoferoles totales (Tabla 3), el contenido de $\gamma$-orizanol es considerablemente superior en todas las muestras: cerca de 20 veces en los salvados de arroz blanco, peletizado y desgrasado, y cerca de 100 veces en el salvado de arroz vaporizado. Gavino et al. (2007) encontraron contenidos de $\gamma$-orizanol en el rango $155-272 \mathrm{mg} / 100 \mathrm{~g}$ en salvado de arroz blanco. Los valores encontrados en este trabajo están dentro de este intervalo (Tabla 5). El salvado desgrasado mostró un contenido de $\gamma$-orizanol un 93,4\% menor que el salvado peletizado. Se deduce que la extracción del aceite con disolventes elimina casi todo el $\gamma$-orizanol (de 192 a 12,7 mg/ $100 \mathrm{~g}$ ).

El $\gamma$-orizanol está también presente en los aceites de otras semillas, como maíz, trigo y cebada, sin embargo, el salvado de arroz es una de las fuentes naturales más abundantes y accesibles
Tabla 5

Concentración de $\gamma$-orizanol en salvados de arroz, ${ }^{a} \mathrm{mg} / 100 \mathrm{~g}$

\begin{tabular}{|c|c|}
\hline Tipo de salvado & $\gamma$-Orizanol $(\mathrm{mg} / 100 \mathrm{~g})$ \\
\hline Arroz blanco & $168 \mathrm{~b}$ \\
\hline Arroz vaporizado & $268 \mathrm{c}$ \\
\hline Peletizado & $192 b$ \\
\hline Desgrasado & $12,7 \mathrm{a}$ \\
\hline
\end{tabular}

${ }^{a}$ Para cada dato de la tabla, $n=6$. Letras distintas indican diferencia significativa al nivel del $5 \%$ de probabilidad para el test de Tukey $(p<0,05)$.

para la obtención de esta mezcla de compuestos (Das et al., 1998). El contenido individual de los distintos componentes del $\gamma$-orizanol en los salvados de arroz investigados (como $\mathrm{mg} / 100 \mathrm{~g}$ de salvado), y su distribución relativa (en porcentajes), se muestran en la Tabla 6.

Con la excepción del componente 2 , el salvado de arroz vaporizado mostró las mayores concentraciones de todos los componentes del $\gamma$-orizanol. El componente 2 fue más abundante en el salvado de arroz blanco. Respecto a los perfiles de los componentes, el componente 4 mostró las mayores concentraciones en todas las muestras investigadas (33,9-35,9\%). Como se ha comentado más arriba, este componente es posiblemente 24-ferulato de metilén-cicloartenilo (Xu y Godber, 1999). También mostraron concentraciones importantes los componentes $5+6(29,0-32,6 \%), 3(13,3-17,5 \%)$ y 7 (11,6-12,1\%). Los demás componentes (1, 2, 8 y 9) aparecieron en todas las muestras con concentraciones muy bajas. En muestras de grano de arroz integral, se encontró que los componentes 3, 4, 5, 6 y 7 del $\gamma$-orizanol eran, por este orden, los de mayor concentración (Engel, 2003), con un perfil cercano al descrito en este trabajo.

\section{CONCLUSIONES}

Se ha verificado que el salvado de arroz vaporizado contiene lípidos en concentración significativamente superior al de los demás tipos de salvado. Respecto a muestra seca, el contenido en lípidos es un $15 \%$ mayor en el salvado de arroz vaporiza-

Tabla 6

Concentración de los principales componentes del $\gamma$-orizanol en salvados de arroz (mg/ $100 \mathrm{~g})$ y su distribución relativa (\%)

\begin{tabular}{|c|c|c|c|c|c|c|c|c|}
\hline \multirow{2}{*}{$\begin{array}{c}\text { Componentes }^{a} \\
1\end{array}$} & \multicolumn{2}{|c|}{ Arroz blanco } & \multicolumn{2}{|c|}{ Arroz vaporizado } & \multicolumn{2}{|c|}{ Peletizado } & \multicolumn{2}{|c|}{ Desgrasado } \\
\hline & 1,70 & 1,0 & 1,89 & 0,7 & 1,76 & 0,9 & 0,25 & 2,0 \\
\hline 2 & 1,46 & 0,9 & 1,28 & 0,5 & 1,17 & 0,6 & 0,17 & 1,3 \\
\hline 3 & 29,4 & 17,5 & 45,0 & 16,8 & 25,4 & 13,3 & 2,19 & 17,2 \\
\hline 4 & 59,2 & 35,1 & 96,0 & 35,9 & 66,5 & 34,7 & 4,31 & 33,9 \\
\hline $5+6$ & 49,4 & 29,3 & 77,5 & 29,0 & 62,5 & 32,6 & 3,71 & 29,2 \\
\hline 7 & 20,3 & 12,1 & 31,9 & 11,9 & 22,8 & 11,9 & 1,48 & 11,6 \\
\hline 8 & 4,36 & 2,6 & 8,61 & 3,2 & 7,34 & 3,8 & 0,36 & 2,8 \\
\hline 9 & 2,47 & 1,4 & 5,34 & 2,0 & 4,30 & 2,2 & 0,23 & 1,8 \\
\hline Total & 168 & 100 & 267 & 100 & 192 & 100 & 12,7 & 100 \\
\hline
\end{tabular}

\footnotetext{
${ }^{a}$ Componentes correspondientes a los picos de la Figura 2B.
} 
do que en salvado de arroz blanco y en salvado peletizado. Además, el menor índice de acidez del salvado de arroz vaporizado pone de manifiesto la eficacia del proceso de vaporización en la estabilización del salvado.

El distinto procesado de los salvados evaluados conduce también a diferencias significativas en el contenido de humedad, si bien, los valores obtenidos son todos muy próximos entre sí, y dependen probablemente de la intensidad de las etapas de secado en cada caso particular.

Respecto al índice de peróxidos, las diferencias no fueron significativas; sin embargo, se observó que los salvados procesados a altas temperaturas, como son el salvado de arroz vaporizado y el salvado peletizado, mostraron índices de peróxidos superiores al del salvado de arroz blanco.

Los distintos tipos de procesado no produjeron en ningún caso alteraciones significativas de la composición porcentual de los ácidos grasos. Los ácidos oleico, linoleico y palmítico fueron mayoritarios en todas las muestras.

Todos los tipos de salvados de arroz mostraron concentraciones importantes de $\alpha$-, $\gamma$ - y $\delta$-tocoferol. Entre éstos, el $\alpha$-tocoferol mostró la mayor concentración en todas las muestras, seguido por el $\gamma$ tocoferol. Asimismo, se observaron hasta nueve componentes del $\gamma$-orizanol en todas las muestras de salvado de arroz. Estos componentes mostraron diferencias importantes de concentración entre muestras de distinto tipo, si bien, los perfiles o concentraciones relativas de unos componentes respecto a otros fueron bastante constantes, no mostrando apenas alteraciones debidas al procesado de los salvados. El salvado de arroz blanco fue el que mostró mayor contenido de tocoferoles $(7,82$ $\mathrm{mg} / 100 \mathrm{~g}$ ), mientras que el salvado de arroz vaporizado fue el que evidenció mayor contenido de $\gamma$ orizanol (268 mg/ $100 \mathrm{~g})$. Finalmente, la etapa de extracción del aceite eliminó cerca de un 91 y un $93 \%$ de los tocoferoles y $\gamma$-orizanol presentes en el salvado, respectivamente.

\section{AGRADECIMIENTOS}

A Irgovel por las muestras de salvado de arroz, al DCA y DCTA de la Universidad Federal de Pelotas, y al CNPQ por el soporte financiero, con colaboración por parte del Proyecto CTQ200761445/BQU (MEC-FEDER, España).

\section{BIBLIOGRAFÍA}

Abidi SL. 2000. Chromatographic analysis of tocol-derived lipid antioxidants. J. Chromatogr. A. 881, 197-216.

AOCS. American Oil Chemists' Society. 1992. Official and tentative methods of the American Oils Chemists' Society, Champaign, IL.

Ausman LM, Rong NI, Nicolosi RJ. 2005. Hypocholesterolemic effect of physically refined rice bran oil: Studies of cholesterol metabolism and early atherosclerosis in hypercholesterolemic hamsters. J. Nutr. Biochem. 16, 521-529.

Block G, Langseth L. 1994. Antioxidant vitamins and disease prevention. Food Technol. 48, 80-84.

Carvalho JLV de, Vieira NR de A. 1999. A cultura do arroz no Brasil: Usos alternativos. Santo Antônio de Goiás: Embrapa Arroz e Feijão, 605-621.

CONAB. 2006. Segundo levantamento de grãos 2006/2007. Disponible en: <http://www.conab.gov.br/ conabweb/download/safra/2levsaf.pdf $>$. Acceso del 26 de noviembre de 2006.

Chen $\mathrm{MH}$, Bergman CJ. 2005. A rapid procedure for analysing rice bran tocopherol, tocotrienol and $\gamma$ orizanol contents. J. Food Anal. 18, 139-151.

Das PK, Chaudhuri A, Kaimal TNB, Bhalerao UT. 1998. Isolation of $\gamma$-oryzanol through calcium ion induced precipitation of anionic micellar aggregates. J. Agric. Food Chem. 46 (8), 3073-3080.

Denardin CC, Silva IP, Silva IP da, Picolli L, Bevilaqua PR, Alves LL, Fagundes CAA. 2003. Composição nutricional do farelo de arroz polido e parboilizado. In: III Congresso brasileiro de arroz irrigado e XXV Reunião da Cultura do Arroz Irrigado, 2003, Balneário Camboriú. Anais do III Congresso brasileiro de arroz irrigado e XXV Reunião da Cultura do Arroz Irrigado, 614-616.

Engel KH, Miller A, Frenzel T, Schmar R. 2003. Coupled liquid chromatography-gas chromatography for the rapid analysis of $\gamma$-oryzanol in rice lipids. $J$. Chromatogr. A 985, 403-410.

Fang N, Yu S, Badger TM. 2003. Characterization of triterpene alcohol and sterol ferulates in rice bran using LC-MS/MS. J. Agric. Food Chem. 51, 32603267.

Gavino VC, Aguilar-Garcia C, Gavino G, BaragañoMosqueda M, Hevia P. 2007. Correlation of tocopherol, tocotrienol, c-oryzanol and total polyphenol content in rice bran with different antioxidant capacity assays. Food Chem. 102, 1228-1232.

Gliszczynska-Swiglo A, Sikorska E. 2004. Simple reversed-phase liquid chromatography method for determination of tocopherols in edible plant oils. J. Chromatogr. A 1048, 195-198.

Gong-Yuanssheng Y, Yao-Huiyuan H. 2001. Purification e identification of gamma-oryzanol from rice bran. J. Chin. Cer. Oils Assoc..16, 30-34.

Hwang DF, Huang SC, Shiau CY, Liu TE, Chu CL. 2005. Effects of rice bran on sensory and physico-chemical properties of emulsified pork meatballs. Meat Sci. 70, 613-619.

Juliano C, Cossu M, Alamanni MC, Piu L. 2005. Antioxidant activity of gamma-oryzanol: mechanism of action and its effect on oxidative stability of pharmaceutical oils. Int. J. Pharmac. 299 (1-2), 146154.

Kim JS, Godber JS, King J, Priyawiwatkul W. 2001. Inhibition of cholesterol autoxidation by the nonsaponifiable fraction in rice bran in an aqueous model system. JAOCS 78, 685-689.

Kitta K, Ebihara M, lizuka T, Yoshikawa R, Isshiki K, Kawamoto S. Variations in lipid content and fatty acid composition of major non-glutinous rice cultivars in Japan. 2005. J. Food Comp. Anal. 18, 269-278.

Lakkakula NR, Lima MH, Walker T. 2004. Rice bran stabilization and rice bran oil extraction using ohmic heating. Biores. Technol. 92, 157-161.

Parrado J, Miramontes E, Jover M, Gutierrez JF, Terán LC de, Bautista J. 2006. Preparation of a rice bran enzymatic extract with potential use as functional food. Food Chem. 98, 742-748. 
Rittner H. 1999. Aspectos críticos da extração de óleos vegetais. Simpósio de óleos e gorduras/ Universidade Federal do Rio Grande. Rio Grande: Furg, 1999.

Rodrigues CEC, Onoyama MM, Meirelles AJA. 2006. Optimization of the rice bran oil deacidification process by liquid-liquid extraction. J. Food Eng. 73 (4) 370-378.

Rodrigues CEC, Pessôa Filho PA, Meirelles AJA. 2004. Phase equilibrium for the system rice bran oil + fatty acids + ethanol + water $+\gamma$-oryzanol + tocols. Fluid Phase Equilibria 216, 271-283.

Silva MA da, Sanches C, Amante ER. 2001. Farelo de arroz composição e propriedades. Óleos \& Grãos 10 (61) 34-42.

Silva MA da, Sanches C, Amante ER. 2006. Prevention of hydrolytic rancidity in rice bran. J. Food Eng. 75 (4) 487-491.

Statsoft. 2004. Inc. Statistica for windows vs. 6.0. Tulsa, OK, USA.

Tortosa E, Barber CB. 1979. El salvado de arroz y su potencial en alimentación animal. Avances en alimentación y mejora animal. Valencia 20 (5) 13-21.
Wilson TA, Nicolosia RJ, Woolfreya B, Kritchevskyb D. 2007. Rice bran oil and oryzanol reduce plasma lipid and lipoprotein cholesterol concentrations and aortic cholesterol ester accumulation to a greater extent than ferulic acid in hypercholesterolemic hamsters. $J$. Nutr. Biochem. 18, 105-112.

Xu Z, Godber JS. 1999. Purification and identification of components of $\gamma$-oryzanol in rice bran oil. J. Agric. Food Chem. 47, 2724-2728.

Zambiazi RC, Przybylsk RI, Zambiazi MW, Mendonça CRB. 2007. Acid composition of vegetable oils and fats. Boletim do CEPPA 25, 111-120.

Zambiazi R. 1997. The role of endogenous lipid components on vegetable oil stability. Manitoba, Canadá, 304f. Tesis (Doctorado en Fisiologia), Food and Nutritional Sciences Interdepartmental Program, University of Manitoba. 